\title{
Microscopic calculation of the temperature anomaly of spatial correlations in liquid ${ }^{4} \mathrm{He}$
}

\author{
R. Blendowske and T. Fliessbach \\ University of Siegen, Fachbereich Physik \\ 59 Siegen, Germany
}

\begin{abstract}
We re-investigate a model of G.V. Chester (Phys. Rev. 100, 455 (1955)) for liquid ${ }^{4} \mathrm{He}$ which combines Jastrow factor correlations with those of the ideal Bose gas. For the first time, we evaluate the structure factor $S(k ; T)$ in this model. This evaluation is based on the optimized random phase approximation frequently used in simple liquid theories. The calculated $S(k ; T)$ reproduces qualitatively the $k$ - and $T$-dependence of the experimental structure factor. The temperature anomaly of liquid helium below the $\lambda$-point can be explained by the Bose gas correlations.
\end{abstract}

PACS: 67.00

Published in J. Phys.: Condens. Matter 4 (1992) 3361-3376 


\section{INTRODUCTION}

Below the $\lambda$-transition the spatial correlations of liquid ${ }^{4} \mathrm{He}$ display an anomalous temperature dependence $[1,2,3]$ : the order increases with temperature. In particular, the main peak of the experimental structure factor $S(k ; T)$ becomes higher and sharper with raising temperature $T$. Close to $T_{\lambda}$ this anomalous temperature dependence is reversed to the normal behaviour.

Microscopically, the spatial correlations of the system are represented by the density matrix. There are basically two approaches for constructing the density matrix, one being related to the ideal Bose gas and the other one to the quasiparticle model.

In this paper we consider a model density matrix which has been proposed by Feynman [4] and Chester [5] for liquid ${ }^{4} \mathrm{He}$. This density matrix combines the Jastrow correlations with those of the ideal Bose gas (IBG). Up to now, the structure factor has not been calculated for this model. We evaluate the structure factor by using techniques which have been developed in the seventies for simple liquids. Before presenting our approach in more detail we shortly review other explanations of the temperature anomaly.

The semi-macroscopic explanation by Cummings et al. [6] assumes a condensate fraction $n_{c}(T)$ with a similar temperature dependence as in the IBG. Qualitatively Cummings et al. argue that the condensate fraction $n_{c}$ is spatially uniform and does, therefore, not contribute to spatial order. Since $n_{c}(T)$ decreases with increasing $T$ the rising number of non-condensed particles is responsible for the increased order. Together with $n_{c}$ the anomalous behaviour disappears at $T_{\lambda}$. In this approach the structure factor $S(k ; T)$ has not been calculated microscopically.

Starting from Landau's quasiparticle picture the microscopic approach by Gaglione et al. [7] relates the anomaly to the enhanced thermal excitations of rotons. A finite roton lifetime is empirically introduced to account for rotonroton interactions. Slightly above $T_{\lambda}$ this leads to an overdamping of the rotons and consequently to a normal behaviour of $S(k ; T)$.

The physical ideas underlying the quasiparticle and the IBG-like approach refer to different temperature regimes. The quasiparticle model requires, in principle, a low quasiparticle density; it is therefore adequate for the low temperature region. When applied to the anomaly problem it has to be extended to higher temperatures, at least to about $2 \mathrm{~K}$. Approaching $T_{\lambda}$, the quasiparticle picture will eventually break down due to the quasiparticle interactions. On the other hand, Feynman [4] argued that around $T_{\lambda}$ the atoms in the liquid should in some essential aspects behave like free particles. He concluded that F. London's [8] famous suggestion about the connection between the $\lambda$-transition and the Bose-Einstein-condensation of the IBG is basically correct.

As already mentioned, we follow the approach based on the ideas of London, Feynman and Chester. Our conceptual starting point is the density matrix of the IBG supplemented by suitable Jastrow functions. The Jastrow functions account for correlations due to the strong repulsion between the atoms. The 
IBG part displays a phase transition which, in this model, can be related to the temperature anomaly. This starting point is specified in Sect. 2.

The structure factor for Chester's density matrix is evaluated in a perturbation scheme. Our scheme is closely related to the optimized random phase approximation (ORPA) used in simple liquid theories. It is described in more detail in Sect. 3.

The decisive temperature dependent correlations are contained in the IBG part of the density matrix. The two particle correlations of the IBG are reviewed in Sect. 4. For the actual calculation a slightly modified IBG is introduced.

Our numerical results for $S(k ; T)$ and their discussion are given in Sect. 5 and 6 , respectively.

\section{DENSITY MATRIX}

The density matrix can be written in the general form:

$$
\rho\left(R, R^{\prime} ; T\right)=\mathcal{N}^{-1} F(R) F\left(R^{\prime}\right) \rho_{M}\left(R, R^{\prime} ; T\right)
$$

Here $R=\left(\mathbf{r}_{1}, \ldots, \mathbf{r}_{N}\right)$ denotes the $3 N$ coordinates of the $N$ atoms contained in a volume $V$. The normalization factor $\mathcal{N}$ is fixed by $\operatorname{Tr} \rho=1$. At zero temperature, $T=0$, the incoherent part becomes $\rho_{M} \equiv 1$, and the factor $F$ reduces to the ground state wave function, $F=\Psi_{\text {g.s. }}$.

The model of Chester [5] is defined by the following assumptions:

- The function $F$ is approximated by a product of temperature independent Jastrow functions,

$$
F(R)=\prod_{i<j}^{N} \exp \left(-\frac{u\left(r_{i j}\right)}{2}\right), \quad r_{i j}=\left|\mathbf{r}_{i}-\mathbf{r}_{j}\right|
$$

This ansatz takes into account the correlations due to the strong repulsion between every pair of atoms; triple and higher correlations are, however, neglected. The Jastrow functions are approximated by those of hard spheres $(h s)$

$$
\exp \left(-u_{h s}(r)\right)=\left\{\begin{array}{lll}
0 & \text { for } & r<d \\
1 & \text { for } & r>d
\end{array}\right.
$$

where $d$ is the $h s$ diameter.

- The temperature dependent incoherent part $\rho_{M}$ is approximated by the density matrix of the ideal Bose gas (IBG),

$$
\rho_{M}=\rho_{\mathrm{IBG}}\left(R, R^{\prime} ; T\right)
$$

Omitting constant factors this density matrix is given by

$$
\rho_{\mathrm{IBG}}\left(R, R^{\prime} ; T\right) \propto \sum_{P} \exp \left(-\frac{\pi}{\lambda_{T}^{2}} \sum_{i=1}^{N}\left[\mathbf{r}_{i}-\mathbf{r}_{P(i)}^{\prime}\right]^{2}\right) .
$$


The summation runs over all $N$ ! permutations $P$ of the $N$ particle indices; the permutation of $(1, \ldots, i, \ldots, N)$ is denoted by $(P(1), \ldots, P(i), \ldots, P(N))$. The quantity $\lambda_{T}=2 \pi \hbar / \sqrt{2 \pi m k_{B} T}$ is the thermal wave length.

The Chester model $(1)-(5)$ is a plausible attempt to combine the attractive features of the IBG (existence of a phase transition) with the strong interactions present in the real liquid. It is not derived from first principles like for example the approaches by Gaglione et al. [7] or by Senger et al. [10]. But an investigation of this model can answer the question wether IBG-related spatial correlations are responsible for the observed anomaly of the structure factor. The techniques for answering this question have been developed in the seventies in the field of simple liquids. By applying these techniques to the Chester model we are able to calculate the structure factor.

The density matrix (1) determines the radial distribution function (rdf) $g(r)$,

$$
g\left(r=r_{12}\right)=\frac{N(N-1)}{\rho_{0}^{2}} \int d^{3} r_{3} \ldots d^{3} r_{N} \rho(R, R)
$$

where $\rho_{0}=N / V$. Its Fourier transform yields the structure factor

$$
S(k)=1+\rho_{0} \int d^{3} r \exp (i \mathbf{k r})[g(r)-1]
$$

For calculating $g$ and $S$ we need the diagonal part of (1) only. The temperature dependence of $S(k)=S(k ; T)$ and other quantities is frequently suppressed in the notation.

Following an idea of Lado [11] we assume the existence of a temperature dependent function $w_{T}(r)$ such that the diagonal part of the IBG density matrix may be replaced by a 'Boltzmann factor':

$$
\rho_{\mathrm{IBG}}(R, R ; T) \approx \prod_{i<j}^{N} \exp \left(-w_{T}\left(r_{i j}\right)\right) .
$$

The approximation of $\rho_{\text {IBG }}$ by a product of two-particle factors is adequate because we restrict ourselves to the evaluation of two-particle correlations, (6) and (7). The exponential form is plausible because for boson systems the density matrix in coordinate space is non-negative. The function $w_{T}$ plays the role of a dimensionless and temperature dependent pseudopotential describing the IBG correlations. It is determined [11] such that the radial distribution functions are the same for both sides of (8):

$$
g\left[\rho_{\mathrm{IBG}}\right] \stackrel{!}{=} g\left[w_{T}\right]
$$

With (8), the diagonal part of the Chester density matrix is given by

$$
\rho(R, R ; T) \approx \mathcal{N}^{-1} \prod_{i<j} \exp \left[-u_{h s}\left(r_{i j}\right)-w_{T}\left(r_{i j}\right)\right]
$$




$$
\mathcal{N}=\int d R \prod_{i<j} \exp \left[-u_{\mathrm{hs}}\left(r_{i j}\right)-w_{T}\left(r_{i j}\right)\right]
$$

The motivation for introducing $w_{T}$ in (8) is that the resulting density matrix (10) can be treated by the diagrammatic methods of simple liquid theories. The function $w_{T}$ is used for intermediate steps only; in the final formulae the IBG correlations are expressed again by $g_{\mathrm{IBG}}$.

\section{PERTURBATION SCHEME}

The pair potential for the atoms in a liquid consists of a strong, short range repulsion and a smoothly varying long range attraction. It is frequently approximated by a $h s$-potential plus a residual interaction $w$. The two-particle correlations of the classical liquid are then represented by Boltzmann factors which are of the same structure as the expression (10). In this expression, the residual interaction $w$ can be treated as a perturbation of the $h s$ system. Suitable schemes for treating this perturbation have been developed in the theory of simple liquids [12].

In the Chester model, the model correlations (4) are weak and long-ranged compared to that of the Jastrow functions (2) and (3). Therefore, we can treat $w_{T}$ as a perturbation of a $h s$ reference system, and adopt the terminology and the methods from the simple liquid theory [12-15]. For evaluating this perturbation we use the optimized random phase approximation (ORPA). Since in our model the $\mathrm{rdf} g_{\mathrm{IBG}}$ is given rather than the corresponding pseudopotential $w_{T}$, we formulate the ORPA in a slightly modified way.

The following derivation relies on the diagrammatic techniques which have been thoroughly investigated in the literature [12-15]. An alternative shorthand derivation of the result (22) is given in App. A.

For defining the ORPA we start with the diagrammatic expansion of the radial correlation function (rcf) $h \equiv g-1$ :

$$
\begin{aligned}
h(1,2)=\{ & \text { all simple diagrams which consist of two white circles } \\
& \text { labelled } 1 \text { and } 2 \text {, black } \rho \text {-circles and } f \text {-bonds, and are } \\
& \text { free of articulation circles }\}
\end{aligned}
$$

Here 1 and 2 stand for $\mathbf{r}_{1}$ and $\mathbf{r}_{2}$, respectively. The dimensionless Fourier transform of $h\left(r_{12}\right)$ is denoted by $T(k)$,

$$
T(k)=S(k)-1=\rho_{0} \int \exp (i \mathbf{k r}) h(r) d^{3} r
$$

The Meyer $f$-function of the whole system is given by

$$
f(r)=\exp \left(-u_{\mathrm{hs}}(r)-w_{T}(r)\right)-1
$$

The Meyer functions of the $h s$ reference and the model system are

$$
f_{0}=\exp \left(-u_{\mathrm{hs}}\right)-1, \quad f_{M}=\exp \left(-w_{T}\right)-1
$$


Eqs. (14) and (15) yield

$$
f=f_{0}+f_{M}+f_{0} f_{M}
$$

This implies that at most $f_{0} f_{M}$-composite diagrams occur in the expansion (12). The summation of the diagrams containing solely $f_{0}$ or $f_{M}$ bonds yield the rcf $h_{0}$ and $h_{M}$, respectively. Applying the concept of a pair of reference or model articulation circles, a topological reduction is performed where all interaction bonds $\left(f_{0}, f_{M}\right)$ are replaced by correlation bonds $\left(h_{0}, h_{M}\right)$. Omitting details of the topological reduction [14] one obtains

$$
h(r)=h_{0}(r)+h_{M}(r)+D\left[h_{0}, h_{M} ; r\right]
$$

where

$$
\begin{aligned}
D=\{ & \text { all at most } h_{0}, h_{M} \text { composite diagrams which consist } \\
& \text { of two white circles, labelled } 1 \text { and } 2 \text {, any number of } \\
& \text { black } \rho \text {-circles, at least one } h_{M^{-}} \text {and one } h_{0} \text {-bond, and } \\
& \text { contain no articulation circles, neither a reference nor } \\
& \text { a model articulation pair of circles }\}
\end{aligned}
$$

The expression (17) with (18) is still exact. The pseudopotential $w_{T}$ has been used for the intermediate steps only; in (17) it has been replaced by the (known) $\operatorname{rcf} h_{M}$.

The random phase approximation (RPA) is equivalent to summing up the subclass of so-called chain diagrams in (18). Using the convolution theorem and requiring $\left|T_{0} T_{M}\right|<1$ this summation yields

$$
\hat{D}_{\mathrm{RPA}}=\left(S_{0}+S_{M}\right) \frac{T_{0} T_{M}}{1-T_{0} T_{M}}
$$

As in (13), the dimensionless Fourier transforms of $h_{0}$ and $h_{M}$ are denoted by $T_{0}=S_{0}-1$ and $T_{M}=S_{M}-1$; the Fourier transforms of all other quantities are marked by hatted symbols. Using $D \approx D_{\mathrm{RPA}}$ in $(17)$ we obtain

$$
h_{\mathrm{RPA}}(r)=h_{0}+h_{M}+D_{\mathrm{RPA}}
$$

and consequently $S_{\mathrm{RPA}}(r)$. Writing

$$
S_{\mathrm{RPA}}(k)=S_{0}(k)+\Delta S_{\mathrm{RPA}}(k)
$$

we find

$$
\Delta S_{\mathrm{RPA}}(k)=T_{M}+\hat{D}_{\mathrm{RPA}}=\frac{T_{M} S_{0}^{2}}{1-T_{0} T_{M}}
$$

Similarily, the radial distribution function becomes

$$
g_{\mathrm{RPA}}(r)=g_{0}(r)+\Delta g_{\mathrm{RPA}}(r)
$$

where $\Delta g_{\mathrm{RPA}}(r)$ is determined by the Fourier transform of $\Delta S_{\mathrm{RPA}}(k)$. 
The result (21) with (22) is applicable to reference systems (index 0) perturbed by model correlations (index $M$ ). In our application the reference system will be the $h s$ system, and the model will be the IBG. We remark that even for model correlations with a diverging Fourier transform $T_{M}(k \rightarrow 0) \rightarrow \infty$ the function $\Delta S_{\mathrm{RPA}}(k)$ remains finite if $S_{0}(k=0)<1$. This corresponds to the effect of repulsive force screening [15].

In the following we recall the arguments given in the classical theory of liquids for the so-called optimization procedure. We consider a $h s$ fluid (3) as a reference system and some perturbation. In an exact perturbation scheme the rdf must vanish inside the hs region. This is expressed by the core condition,

$$
g(r<d) \equiv 0
$$

This condition is trivially fulfilled for the $h s$ system, $g_{0} \equiv 0$. Applied to (23) the core condition reads

$$
\Delta g_{\mathrm{RPA}}(r<d) \equiv 0 .
$$

In general, this condition will be violated due to the approximation $D \approx D_{\mathrm{RPA}}$ used for $\Delta g_{\mathrm{RPA}}$. The optimization procedure is a method of healing this deficiency.

In a valid perturbation scheme for a $h s$ reference system, the result must be independent of the functional form of the perturbation in the region $r<d$ (we exclude singular functions). We are therefore free to replace $h_{M}(r)$ in (17) by

$$
\widetilde{h}_{M}=\left\{\begin{array}{c}
h_{M}(r) \quad \text { for } \quad r>d \\
\sum_{n} a_{n} f_{n}(r) \text { for } r<d
\end{array}\right.
$$

Here $\left\{f_{n}(r)\right\}$ is, in principle, a complete set of basis functions. The optimization procedure [12] consists now in determining the $a_{n}$ such that the core condition is fulfilled. Calculations in classical liquids have shown that the optimization procedure is decisive for obtaining valid results [12]; this is also confirmed by our calculations.

For our purpose, it turns out that the set of five functions, $f_{n}(r)=r^{n}$ with $n=0, \ldots, 4$ forms a sufficient basis. The coefficients $a_{n}$ are determined by minimizing the positive definite functional

$$
F=\int d^{3} k \frac{S_{0}}{T_{0}^{2}}\left[\ln \left(1-T_{0} \widetilde{T}_{M}\right)+T_{0} \widetilde{T}_{M}\right]
$$

The functional derivative of $F$ yields $\Delta S_{\mathrm{RPA}}=\delta F / \delta \widetilde{T}_{M}$. For minimizing the deviations from (25) the conditions $\partial F / \partial a_{n}=0$ are required [12].

The optimized results are denoted by

$$
S_{\mathrm{ORPA}}(k)=S_{0}(k)+\Delta S_{\mathrm{ORPA}}(k)=\frac{\widetilde{T}_{M} S_{0}^{2}}{1-T_{0} \widetilde{T}_{M}}
$$


and by $g_{\mathrm{ORPA}}(r)=g_{0}(r)+\Delta g_{\mathrm{ORPA}}(r)$, respectively.

In order to test our perturbation scheme we performed standard Monte-Carlo (MC) calculations for (10). For this calculation, the IBG pseudopotential $w_{T}(r)$ has been determined numerically via the BBGKY equation [11] for $T \geq T_{\lambda}$; we recall at this point that $w_{T}$ is not needed for (28). The $\mathrm{MC}$ calculations have been carried out for $N=128$ particles, $3 \times 10^{6}$ configurations and periodic boundary conditions. The result $\Delta g_{\mathrm{MC}}(r)$ agrees with $\Delta g_{\mathrm{ORPA}}(r)$ within $15 \%$ in the range $3.05 \AA \leq r \leq 3.95 \AA$. This value is quite acceptable for our purpose. For $r>4.0 \AA$ the effects are overlayed by the 'noise' of the MC-results which is of the order of 1\%. This noise makes a unique Fourier transform difficult; therefore a standard MC method can not replace our perturbation scheme.

\section{MODEL CORRELATIONS}

The two-particle correlations of the IBG have been derived many times $[11,16-$ 18]. For $N$ free Bose particles in a volume $V$ the $\operatorname{rdf}$ is given by

$$
g_{\mathrm{IBG}}(r ; T)=1+\frac{1}{N^{2}}\left(\sum_{\mathbf{k}} \frac{\sin (k r)}{k r} n(k)\right)^{2}-\sum_{\mathbf{k}} \frac{n(k)^{2}}{N^{2}}-\frac{1}{N}
$$

The single particle energies $\varepsilon=\hbar^{2} k^{2} / 2 m$ determine the average occupation numbers

$$
n(k)=n(\mathbf{k})=\frac{1}{\exp \left((\varepsilon-\mu) / k_{B} T\right)-1}=\frac{1}{\exp \left(x^{2}+\tau^{2}\right)-1}
$$

This formula is valid for the non-condensed particles. In the last expression we have introduced the dimensionless quantities

$$
\tau^{2}=-\frac{\mu}{k_{B} T}, \quad x^{2}=\frac{\varepsilon(k)}{k_{B} T}=\frac{\hbar^{2} k^{2}}{2 m k_{B} T}=\frac{\lambda_{T}^{2}}{4 \pi} k^{2}
$$

The chemical potential $\mu$ or, equivalently, $\tau$ is fixed by the particle number condition $N=\sum n(k)$. This condition leads to a transition temperature $T_{\mathrm{c}}$ below which the Bose-Einstein-condensation sets in. This means that a finite fraction $n_{0}=\mathcal{O}(1)$ of all particles occupies the lowest single particle state,

$$
n_{0}=\frac{n\left(k_{0}\right)}{N}=1-\sum_{\mathbf{k} \neq \mathbf{k}_{0}} \frac{n(k)}{N}
$$

In a given (macroscopic) volume $V$ the lowest possible wave vector $\mathbf{k}_{0}$ is not exactly equal to zero. For $T \rightarrow T_{\mathrm{c}}$ the particle number condition yields $\mu-$ $\varepsilon\left(k_{0}\right) \rightarrow 0$. For simplicity we rename $\mu-\varepsilon\left(k_{0}\right)$ by $\mu$. Then $\mu$ and $\tau$ vanish at $T_{\mathrm{c}}$. The transition temperature $T_{\mathrm{c}}$ is given by

$$
\lambda_{T_{\mathrm{c}}}=\frac{2 \pi \hbar}{\sqrt{2 \pi m k_{B} T_{\mathrm{c}}}}=\left(v \zeta_{3 / 2}\right)^{1 / 3}
$$


where $v=V / N$ and $\zeta_{3 / 2} \approx 2.6124$ denotes Riemann's zeta function. We identify $T_{\mathrm{c}}$ with the experimental temperature $T_{\lambda}$ by assuming that $m$ in (31) is an effective mass [4].

Neglecting terms of the order $1 / N$ the IBG rdf reads

$$
g_{\mathrm{IBG}}(r ; T)=1+Q^{2}\left(r / \lambda_{T}\right)+2 n_{0} Q\left(r / \lambda_{T}\right)
$$

where

$$
Q(y)=\frac{v}{\lambda_{T}^{3}} \sum_{n=1}^{\infty} \frac{\exp \left(-n \tau^{2}-\pi y^{2} / n\right)}{n^{3 / 2}}
$$

For $y \gg 1$ the function $Q$ decays exponentially for $\tau \neq 0$ and like $1 / y$ for $\tau=0$. For $k \rightarrow 0$ the structure factor $S_{\mathrm{IBG}}$ diverges then like $1 / k$ for $T=T_{\lambda}$, and like $1 / k^{2}$ for $T<T_{\lambda}$.

In the following we introduce a slightly modified IBG which leads to a more realistic temperature dependence of thermodynamic properties and of $n_{0}(T)$. We expand $\tau$ for small relative temperatures $t=\left(T-T_{\lambda}\right) / T_{\lambda}$,

$$
\tau=\left\{\begin{array}{rll}
a t+\mathcal{O}\left(t^{2}\right) & \text { for } & t \geq 0 \\
a^{\prime}|t|+\mathcal{O}\left(t^{2}\right) & \text { for } & t<0
\end{array}\right.
$$

This expansion yields

$$
n_{0}=\left\{\begin{array}{cc}
0 & \text { for } t \geq 0 \\
f|t|+\mathcal{O}\left(t^{2}\right) & \text { for } t<0
\end{array}\right.
$$

for the model condensate fraction $n_{0}$. The particle number condition yields $a=3 \zeta_{3 / 2} /(4 \sqrt{\pi})$ and relates $f$ to $a^{\prime}$,

$$
f=\frac{3}{2}+\frac{2 \sqrt{\pi}}{\zeta_{3 / 2}} a^{\prime}
$$

In the IBG one has $\tau \equiv 0$ for $t<0$ and therefore $a^{\prime}=0$. Deviating from the IBG the modified IBG uses $\tau=a^{\prime}|t|$ with $a^{\prime} \neq 0$. Physically $a^{\prime} \neq 0$ implies a temperature dependent energy gap $\Delta=a^{\prime 2} t^{2} k_{B} T$ for the macroscopically occupied single particle state; this kind of modification has already been considered in Ref. [19]. The energy gap leads to a faster $(f>3 / 2)$ occupation of the condensed state for decreasing temperature.

The introduced modification of the IBG has no microscopic justification but it can be motivated by the following reasons. First of all, the form of (36) is plausible to some extent because of its simplicity and symmetry. Secondly, it removes two shortcomings of the IBG with respect to liquid ${ }^{4} \mathrm{He}$ : the divergence of the structure factor for $k \rightarrow 0$ and $t<0$, and the unrealistic slow decay of the specific heat $\left(\propto T^{3 / 2}\right)$. Similarily as in the roton picture, the energy gap $\Delta=a^{\prime 2} t^{2} k_{B} T$ leads to an exponential decay of the specific heat and therefore to a better overall agreement in the temperature region $1 \mathrm{~K}<T<2 \mathrm{~K}$. 
Within the framework of the IBG, the model condensate fraction $n_{0}$ equals the superfluid fraction $\rho_{s} / \rho_{0}$. The historical experimental fit formula [20]

$$
\rho_{S} / \rho_{0}=1-(1+|t|)^{f}, \quad f=5.6
$$

can be fairly well reproduced by $n_{0}(T)$ of (32) if this $f=5.6$ is identified with the $f$ of (38). This yields $a^{\prime}=3.0$.

The expression (39) describes the data roughly in the range between 1 and $2 \mathrm{~K}$; it is qualitatively wrong in the critical region and in the phonon dominated region. In this respect the IGB as well as the modified one fail. For $T=1 \ldots 2 \mathrm{~K}$ it is, however, possible to identify $n_{0}$ with $\rho_{s} / \rho_{0}$ if $a^{\prime} \neq 0$ is admitted. In Ref. [21] the relation between $n_{0}$ and $\rho_{s} / \rho_{0}$ is investigated in more detail including the critical region; this leads to the same value $a^{\prime}=3.0$. It should be noted that the model condensate fraction $n_{0}$ defined by (32) is not identical to what is usually called condensate fraction $n_{c}$; in particular $n_{0}=1$ for $T=0$ is depleted by the Jastrow factors to about $n_{c} \approx 10 \%$.

Having fixed the value of $a^{\prime}$ we may express the assumed modification by

$$
a^{\prime}=\left\{\begin{array}{cc}
0 & \text { IBG } \\
3.0 & \text { modified IBG }
\end{array}\right.
$$

We emphasize that this is indeed a minor modification. All formulae displayed in this section are valid for the IBG as well as for the modified IBG. For $T \geq T_{\lambda}$ the IBG and the modified IBG coincide. The structure of the spatial correlations in the IBG are determined by the many-body symmetry entering the calculation of the rdf; this structure is not affected by $a^{\prime} \neq 0$. The parameter $a^{\prime}$ has, however, an influence on the temperature dependence of these correlations.

We conclude this section by a discussion of the range of validity of the model correlations. The model does not include phonons which are dominant below $1 \mathrm{~K}$; therefore we have to restrict ourselves to $T>1 \mathrm{~K}$. In the region 1 to $2 \mathrm{~K}$ the modified IBG reproduces fairly well the thermodynamic properties and should therefore contain the relevant temperature dependent correlations. For $T \approx T_{\lambda}$ the model displays a phase transition and should therefore contain at least part of the relevant correlations. The model does, however, not describe the specific correlations causing the logarithmic singularity; the temperature dependence of the specific heat indicates that these correlations are dominant for $|t| \leq 0.01$ and still important for $|t| \leq 0.1$. Summarizing, the model correlations might be quantitatively right between 1 and $2 \mathrm{~K}$, and qualitatively right across the phase transition.

\section{$5 \quad$ RESULTS}

The ORPA result (28) together with the model correlations specified in Sect. 4 defines our theoretical structure factor,

$$
S_{\text {theor }}(k ; T)=S_{\text {theor }}(k)+\Delta S_{\text {theor }}(k ; T)
$$


where

$$
S_{\text {theor }}(k)=S_{0}(k), \quad \Delta S_{\text {theor }}(k ; T)=\frac{\widetilde{T}_{M} S_{0}^{2}}{1-T_{0} \widetilde{T}_{M}} \quad\left(\begin{array}{l}
0: h s \text {-model system } \\
M: \text { modified IBG }
\end{array}\right)
$$

This theoretical expression is compared to the experimental structure factor

$$
S_{\exp }(k ; T)=S_{\exp }(k ; 1 \mathrm{~K})+\Delta S_{\exp }(k ; T)
$$

Our model is expected to describe the relevant temperature dependent effects above $1 \mathrm{~K}$. Therefore we adjust the parameters of our $h s$ reference system to the experimental structure factor at $1 \mathrm{~K}$. This yields a $h s$ diameter $d=3.0 \AA$ and a packing fraction of $\eta=0.26$. As usual, the structure factor $S_{0}(k)=S_{\mathrm{hs}}(k)$ is determined by the PY equation [12].

In Fig. 1 the calculated result $S_{\text {theor }}=S_{\text {hs }}$ is compared to $S_{\exp }(1 \mathrm{~K})$. The overall agreement shows that the experimental structure factor can indeed be simulated by the one of a classical $h s$ fluid with suitable parameters. Of course, this is not a theory for $S(k ; 1 \mathrm{~K})$. For a determination of $S(k ; 1 \mathrm{~K})$ from first principles [22] one has to take into account quantum mechanical effects, the phonon contributions [23] and the attractive part of the interaction.

Our aim is the determination of the additional temperature dependent effects due to the IGB related correlations. For this purpose we need a reference system. The optimization procedure requires in particular a $h s$ reference system; this means that in a consistent treatment we cannot use $S_{\exp }(k ; 1 \mathrm{~K})$ instead of $S_{\mathrm{hs}}$. For such a reference system the agreement in Fig. 1 is quite satisfactory.

The temperature dependent part $\Delta S_{\text {theor }}(k ; T)$ of the theoretical structure factor is determined by $T_{0}=S_{0}-1$ and $\widetilde{T}_{M}$. Here $T_{0}$ refers to the $h s$ reference system, and $\widetilde{T}_{M}$ is the Fourier transform of $\widetilde{h}_{M}$. The function $\widetilde{h}_{M}$ is the optimized model $\operatorname{rcf} h_{M}$. The model correlations are that of the modified IBG; they are defined by (34) and (35). Details of the numerical evaluation of the model correlations are given in App. B. We use the experimental values for the number density $\rho_{0}=N / V=0.022 \AA^{-3}$ and for the transition temperature $T_{\lambda}=2.17 \mathrm{~K}$.

Fig. 2 presents the results for $\Delta S_{\text {theor }}\left(k ; T_{\lambda}\right)$ with and without optimization. The large difference between these two functions demonstrates the crucial role of the core condition. The result without optimization is unphysical because the corresponding $\Delta g_{\text {theor }}(r)$ does not vanish for $r<d$.

In Fig. 3 the $k$-dependence of $\Delta S_{\text {theor }}$ is compared to $\Delta S_{\exp }$ for two temperature values. The model prediction $\Delta S_{\text {theor }}$ reproduces the $k$-dependence of $\Delta S_{\text {exp }}$ remarkably well. It yields a central peak at about the right position, and describes the oscillations and the $k \rightarrow 0$ behaviour fairly well. The position $k_{\text {max }} \approx 2 \pi / d$ of the main peak is essentially determined by the $h s$ diameter $d$; it is not a specific property of the model correlations. We remark that the plateau of $\Delta S_{\exp }$ around $k \approx 0.8 \AA^{-1}$ is an artefact. It stems from the connection of neutron scattering data [2] for larger $k$-values and x-ray data for smaller $k$-values, see Fig. 14 of Ref. [3]. 
The absolute values of $\Delta S_{\text {theor }}$ are too small by a factor of 2 to 3 . This discrepancy is outside the range of experimental uncertainties of about \pm 0.01 for the absolute value of $\Delta S_{\exp }$. (The errors of $S_{\text {exp }}$ itself are estimated to be smaller than $1 \%$ [2].) It is also outside the range of the errors due to the approximations used for the evaluation in the given model; these errors are about $15 \%$ for the ORPA (last paragraph of section 4) and about 10\% due to the uncertainty in the parameters of the $h s$ reference system. The discrepancy in the absolute size of the effect indicates that part of the correlations is missing in our model. One might expect that these are the specific correlations responsible for the logarithmic singularity; they are expected to yield a major contribution in the range $|t|<0.1$.

The model prediction $\Delta S_{\text {theor }}$ reproduces qualitatively also the temperature dependence of $\Delta S_{\text {exp }}$, Fig. 3. As a function of temperature, $\Delta S_{\text {theor }}$ has its maximum value slightly below $T_{\lambda}$, it decreases with decreasing temperature, and disappears below $T \simeq 1.3 \mathrm{~K}$. These properties agree with that of the experimentally observed anomaly. The decay of $\Delta S_{\text {theor }}$ with decreasing temperature is, however, too slow. The central peak of $\Delta S_{\text {theor }}$ reaches its half value at $T=1.74 \mathrm{~K}$ compared to roughly $T \approx 1.9 \mathrm{~K}$ for $\Delta S_{\text {exp }}$. The decay of $\Delta S_{\text {theor }}$ depends on the value of the parameter $a^{\prime}$. At $T_{\lambda}$ the IBG and the modified IBG coincide. For the pure IBG $\left(a^{\prime}=0\right)$ the decay sets in at a lower temperature and is less pronounced. On the other hand, a larger value of $a^{\prime}$ makes the decay steeper. We prefer not to use $a^{\prime}$ as a fit parameter for this detail because the too slow fall-off is probably connected to the missing critical correlations.

There is a corresponding decay of $\Delta S_{\text {theor }}$ for increasing temperature above $T_{\lambda}$; the $k$ - and $|t|$-dependence is similar for $t<0$ and $t>0$. The reproduction of $\Delta S_{\exp }$ for $T>T_{\lambda}$ is of the same quality as that shown in Fig. 3.

Summarizing we state that the anomaly can be qualitatively explained by the correlations of the modified IBG. We emphasize that all parameters of the model are fixed by experimental quantities which are independent of $\Delta S_{\exp }$.

\section{DISCUSSION}

The model proposed by Chester is the most simple but realistic ansatz for the density matrix of ${ }^{4} \mathrm{He}$ with a phase transition at $T_{\lambda}$. In this model, the main features of the considered anomaly are reproduced, in particular its $k$ dependence, its temperature range and its reversal close to $T_{\lambda}$.

The model fails in reproducing the absolute values of $\Delta S_{\text {exp }}$ by a factor of 2 to 3. This is not too surprising because the entropy of the IBG (modified or not) at $T_{\lambda}$ is 1.7 times higher than the experimental one. This means that in liquid helium the particles are more ordered or correlated than in the IBG model. The missing correlations might be that which are responsible for the logarithmic singularity at $T_{\lambda}$.

Around $k \approx 2 \AA^{-1}$ the results by Gaglione et al. [7] are quantitatively superior to ours. Their results depend, however, on the empirical expression for 
roton life times. Our results are, on the other hand, essentially fixed by the IBG correlations. The underlying physical ideas are quite different: Gaglione et al. use the quasiparticle model where an overdamping of the rotons leads to the reversal of the temparature anomaly. We use the IBG plus Jastrow correlations where the anomaly is related to the phase transition of the IBG.

Discussing the approach by Cummings et al. [6], Gaglione et al. proposed to measure $S(k ; T)$ along different isochores. In the quasiparticle model one expects that the anomaly effect increases with the density. In the model of Cummings et al. a decrease is expected because all theories predict a reduction of the condensate fraction $n_{c}$ with enhanced density. In our description the anomaly is related to the model condensate fraction, but its effect on $S(k)$ is decisively influenced by the reference system (in particular, by the core condition). For a higher density the effect of the core condition will increase. Preliminary calculations show a slight increase of $\Delta S_{\text {theor }}$ with the density leading to a qualitative agreement with Gaglione et al..

The otherwise successfull variational ab initio calculations of Senger et al. [10] display neither a phase transition at $T_{\lambda}$ nor a reversal of the temperature anomaly. From our point of view, their ansatz for the density matrix should be supplemented by IBG related correlations.

The path-integral computations of Ceperley and Pollock [24] are in good agreement with the experimental $\operatorname{rdf} g(r ; T)$ down to $1 \mathrm{~K}$. The results for $g(r ; T)$ contain some indication of an anomalous behaviour. The structure factor $S(k ; T)$ in which the anomaly effect is more obvious has, however, not been evaluated.

The exchange symmetry plays a decisive role for the $\lambda$-transition; this follows from the absence of this transition in liquid ${ }^{3} \mathrm{He}$. The IBG is the simplest model which takes the symmetry effects fully into account. The IBG yields a phase transition which exhibits a number of similarities to the $\lambda$-transition; moreover, the unusual hydrodynamic properties of He II can be understood in this model [25]. In some respects the IBG is similar to the nuclear shell model which is a quite successfull ideal gas model for the strongly interacting nuclear liquid. Both ideal gas models are, of course, unable to reproduce the two-particle correlations caused by the interaction. A simple remedy of this failure is to supplement these models by Jastrow factors. For the IBG this leads to the Chester model in which an at least semi-quantitative description of the two-body correlations is possible. As we have demonstrated this model is able to explain the temperature anomaly. This success might encourage renewed investigations of IBG related models.

\section{APPENDICES}

\section{A RPA STRUCTURE FACTOR}

In this appendix we present a shorthand derivation of the main result (21) of Sect. 3 without using diagrammatic techniques. 
We consider a reference system with the structure factor $S_{0}$ and a perturbing (dimensionless) potential $w(r)$. The structure factor $S$ of the whole system can be written as [12]

$$
S=\frac{S_{0}}{1-\left(\hat{c}-\hat{c}_{0}\right) S_{0}}
$$

Here $\hat{c}$ and $\hat{c}_{0}$ are the Fourier transforms of the direct correlation functions for the whole and the reference sytem, respectively. They are defined by the Fourier transform of the Ornstein-Zernicke relation,

$$
\hat{c}(k)=\frac{T(k)}{S(k)}
$$

The canonical way of introducing the RPA is

$$
c-c_{0} \approx-w
$$

Using the linearized HNC-equation one obtains

$$
c_{M} \approx-w
$$

Combining the last two equations and using (45) for $\hat{c}_{M}$ we get

$$
\hat{c}(k)-\hat{c}_{0}(k) \approx \hat{c}_{M}(k)=\frac{T_{M}(k)}{S_{M}(k)}
$$

We insert this in (44),

$$
S_{\mathrm{RPA}}(k)=\frac{S_{0}}{1-S_{0} T_{M} / S_{M}}=S_{0}+\frac{T_{M} S_{0}^{2}}{1-T_{0} T_{M}}
$$

This is the wanted result.

\section{B EVALUATION OF IBG CORRELATIONS}

In this appendix we describe the numerical evaluation of the structure factor and the rdf of the IBG. All formulae apply equally well to the modified IBG.

For $N$ spinless bosons the structure factor is given by

$$
S_{M}(q)=1+T_{M}(q)=\frac{1}{N} \sum_{\mathbf{p}, \mathbf{p}^{\prime}}\left\langle a_{\mathbf{p}^{\prime}-\mathbf{q}}^{+} a_{\mathbf{p}^{\prime}} a_{\mathbf{p}+\mathbf{q}}^{+} a_{\mathbf{p}}\right\rangle
$$

The operators $a_{\mathbf{p}}^{+}, a_{\mathbf{p}}$ create or annihilate a particle in the state with momentum $\mathbf{p}$. They obey the commutation rule $\left[a_{\mathbf{p}}^{+}, a_{\mathbf{p}}\right]=\delta_{\mathbf{p}, \mathbf{p}^{\prime}}$. The brackets in (50) denote the quantum mechanical expectation value for the many-body states $|\ldots, \nu(\mathbf{p}), \ldots\rangle$ and the statistical expectation value. The statistical average is effectively performed by replacing the occupation numbers $\nu(\mathbf{p})$ by their expectation values $\langle\nu(\mathbf{p})\rangle=n(\mathbf{p})$. The average occupation numbers $n(\mathbf{p})$ are given by $(30)$ and $(32)$. 
Separating off the condensed particles, $S_{M}(q)$ becomes

$$
S_{M}(q)=1+2 \frac{n\left(k_{0}\right)}{N} n(q)+\frac{1}{N} \sum_{\mathbf{p} \neq \mathbf{k}_{0}} n(\mathbf{p}) n(\mathbf{p}+\mathbf{q})=1+T_{\mathrm{c}}+T_{\mathrm{nc}}
$$

As in Sect. 3, $k_{0}$ denotes the lowest possible single particle state. The part $T_{\mathrm{c}}$ is determined by (30) and (32). For evaluating the contribution $T_{\mathrm{nc}}$ of the noncondensed particles we introduce polar coordinates and replace the summation by an integration:

$$
T_{\mathrm{nc}}=\frac{v}{4 \pi^{2}} \int_{-\infty}^{+\infty} d k^{\prime} \int_{0}^{\infty} d k^{\prime \prime} n\left(\sqrt{k^{\prime 2}+k^{\prime \prime 2}}\right) n\left(\sqrt{k^{\prime \prime 2}+\left(k^{\prime}+q\right)^{2}}\right)
$$

The wave vector $\mathbf{k}$ is written as $\mathbf{k}=\mathbf{k}^{\prime}+\mathbf{k}^{\prime \prime}$ with $\mathbf{k}^{\prime \prime} \mathbf{q}=0$, and $v=V / N$ denotes the volume per particle. We insert the average occupation numbers (30) and (32), use the quantities

$$
\alpha\left(k^{\prime}\right)=\exp \left(-\delta k^{\prime 2}\right), \quad \beta\left(k^{\prime}\right)=\exp \left(-\delta\left(k^{\prime 2}+q^{2}\right)\right), \quad \delta=\frac{\lambda_{T}^{2}}{4 \pi}, \quad z=\exp \left(-\tau^{2}\right)
$$

and perform the integration over $k^{\prime \prime}$ :

$$
T_{\mathrm{nc}}=-\frac{v}{8 \pi^{2} \delta} \int_{-\infty}^{\infty} d k^{\prime} \frac{\alpha-\beta}{\alpha \beta}\left[\frac{\ln (1-z \alpha)}{\alpha}-\frac{\ln (1-z \beta)}{\beta}\right]
$$

We replace $k^{\prime}$ by $k^{\prime}-q / 2$, and introduce $y=2 \delta q k^{\prime}$ and $\gamma=q \sqrt{\delta}$. This yields

$$
T_{\mathrm{nc}}(q)=-I-\frac{v}{\lambda_{T}^{4}} \frac{1}{q} \int_{0}^{\infty} d y \frac{1+\exp (-y)}{1-\exp (-y)} \ln \left(\frac{1-z B_{-}(y)}{1-z B_{+}(y)}\right)
$$

where

$$
B_{ \pm}(y)=\exp \left(-\frac{\left(y \pm \gamma^{2}\right)^{2}}{4 \gamma^{2}}\right)
$$

and

$$
I=1-\frac{n\left(k_{0}\right)}{N}=-\frac{v}{\left(2 \pi \lambda_{T}\right)^{2}} \int_{-\infty}^{\infty} d k^{\prime} \ln \left[1-z \exp \left(-\delta k^{\prime 2}\right)\right]
$$

The integrals in (55) and (57) are evaluated numerically. The substitution $\pm u^{2}=$ $y-\gamma^{2}$ makes the logarithmic singularity at $y=0$ numerically integrable. The integrals remain finite for $\tau \rightarrow 0$ and $q \rightarrow 0$ because the $1 / q$-factor has been split off. This completes the evaluation of the structure factor.

For the model rdf (34) we have to compute the function $Q(r)$ which can be expressed by the sum (35) or by the integral

$$
Q(r)=\frac{v}{2 \pi^{2}} \frac{1}{r} \int_{0}^{\infty} d k \frac{k \sin k r}{\exp \left(\tau^{2}+\delta k^{2}\right)-1}
$$


For small values of $r$ this integral is solved numerically. For large $r$, say $r / \lambda_{T}>1$, this is no longer possible because the integrand oscillates too rapidly. We use then the expansion

$$
Q(r)=\frac{v}{\lambda_{T}^{3}}\left(\frac{1}{x} \exp (-2 \sqrt{\pi} \tau x)+2 \sum_{m \geq 1} \exp \left(-A_{m}^{+} x\right) \cos \left(A_{m}^{-} x\right)\right)
$$

where

$$
A_{m}^{ \pm}=\sqrt{2 \pi}\left(\tau^{4}+4 \pi^{2} m^{2}\right)^{1 / 4}\left[1 \pm \frac{\tau^{2}}{\left(\tau^{4}+4 \pi^{2} m^{2}\right)^{1 / 4}}\right]^{1 / 4}
$$

and $x=r / \lambda_{T}$. This expansion may be found in Ref. [16], eq. (2.24).

\section{References}

[1] E. C. Svensson and V. F. Sears, Physica 137 B, 126 (1986)

[2] E. C. Svensson, V. F. Sears, A. D. B. Woods and P. Martel, Phys. Rev.

B 21, 3638 (1980)

[3] H. N. Robkoff and R. B. Hallock, Phys. Rev. B 24, 159 (1981)

[4] R. P. Feynman, Phys. Rev. 91, 1291 (1953)

[5] G. V. Chester, Phys. Rev. 100, 455 (1955)

[6] F. W. Cummings, G. F. Hyland and R. Rowlands, Phys. Lett. A 86, 370 (1981)

[7] G. Gaglione, G. L. Masserini and L. Reatto, Phys. Rev. B 23, 1120 (1981)

[8] F. London, Nature 141, 643 (1938)

[9] W. L. McMillan, Phys. Rev 138, 442 (1965)

[10] G. Senger, M. L. Ristig, K. E. Krten, C. E. Campbell, Phys. Rev. B 33, 7562 (1986)

[11] F. Lado, J. Chem. Phys. 47, 5369 (1967)

[12] J. R. Hansen, I. R. McDonald, Theory of Simple Liquids, 2nd edition, Academic Press 1986

[13] I. R. McDonald, S. P. O'Gorman, Phys. Chem. Liq. 8, 57 (1978)

[14] H. C. Anderson, D. Chandler, J. Chem. Phys. 57, 1918 (1972) 
[15] H. C. Anderson, D. C. Chandler, J. D. Weeks, Adv. Chem. Phys. 34, 105 (1976)

[16] R. M. Ziff, G. E. Uhlenbeck, M. Kac, Phys. Reports 32, 169 (1977)

[17] F. London, J. Chem. Phys. 11, 203 (1943)

[18] J. K. Jaen, Journ. Math. Phys. 8, 1438 (1967)

[19] A. Bijl, J. de Boer, A. Michels, Physica 8, 655 (1941)

[20] F. London, Superfluids, Vol. II, Dover Publications, Inc., New York 1954

[21] T. Fliessbach, Nuovo Cimento D 13, 211 (1991)

[22] C. C. Chang and C. E. Campbell, Phys. Rev. B15, 4238 (1977)

[23] L. Reatto, G. V. Chester, Phys. Rev. 155, 88 (1966)

[24] D. M. Ceperley, E. L. Pollock, Phys. Rev. Lett. 56, 351 (1986)

[25] S. J. Putterman, Superfluid Hydrodynamics, North Holland Publ. Comp., London 1974 


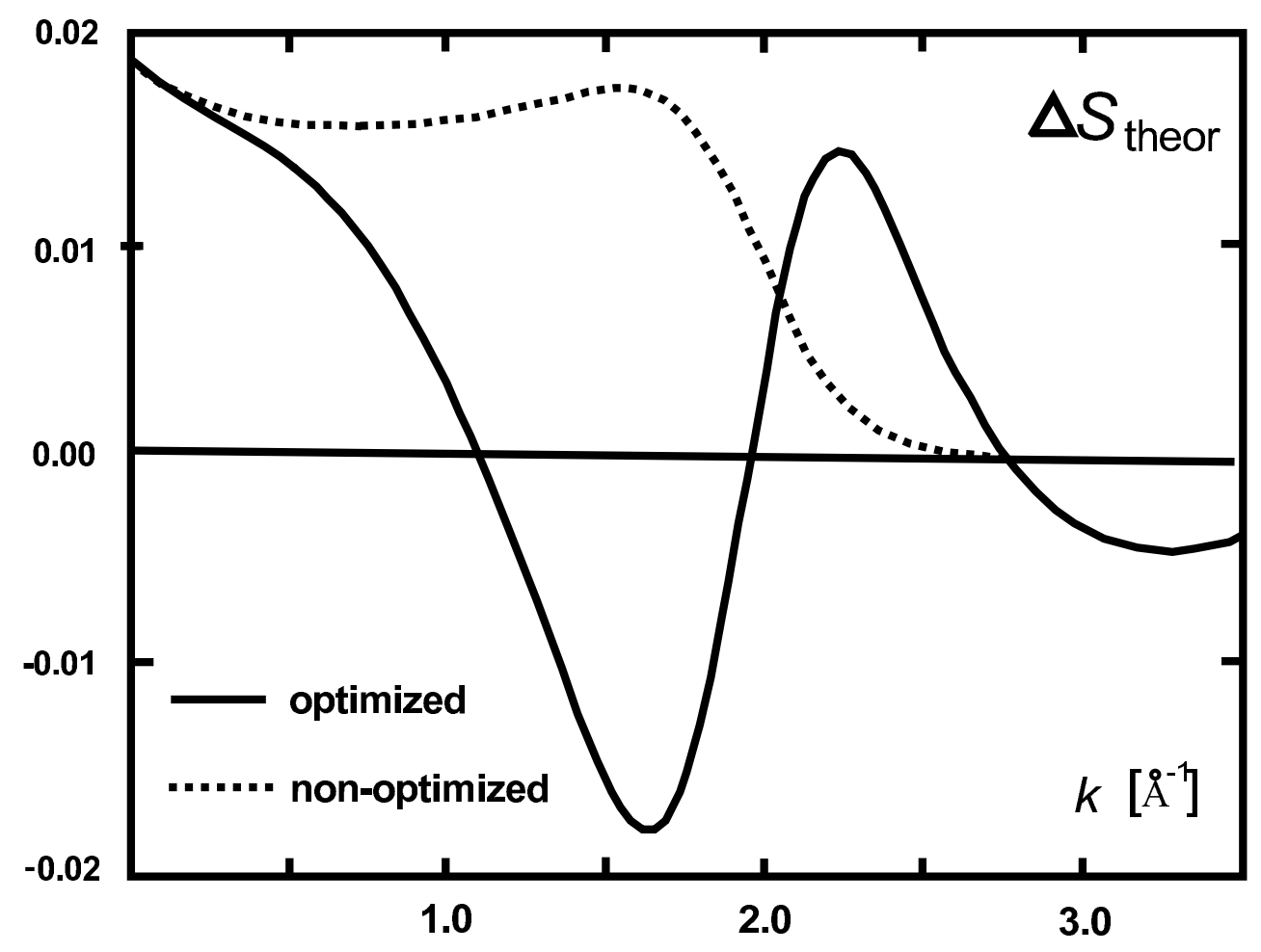

Figure 1: The experimental structure factor $S_{\exp }(k ; 1.0 \mathrm{~K})([2]$, dashed line) is compared to the structure factor $S_{\mathrm{hs}}(k)$ (full line) of a hard sphere system. Since the $h s$ model serves as the reference system its parameters (hard sphere diameter and packing fraction) are adjusted in order to minimize the deviations. 


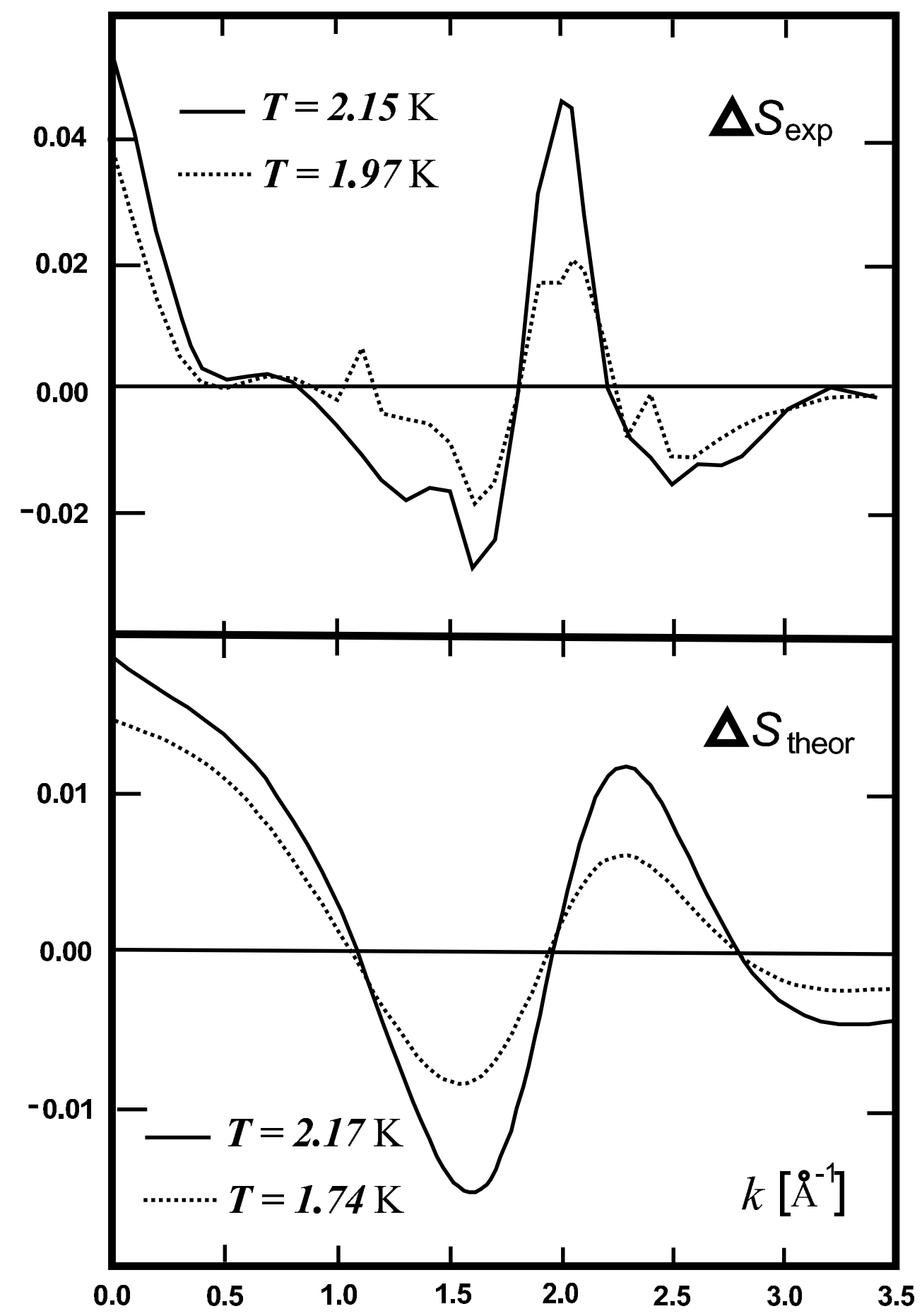

Figure 2: The non-optimized result (dotted line) for $\Delta S_{\text {theor }}\left(k ; T_{\lambda}\right)$ is compared to the optimized one (full line). Only the optimized result meets the physical core condition. The drastic differences show that the optimization procedure is indispensable. 


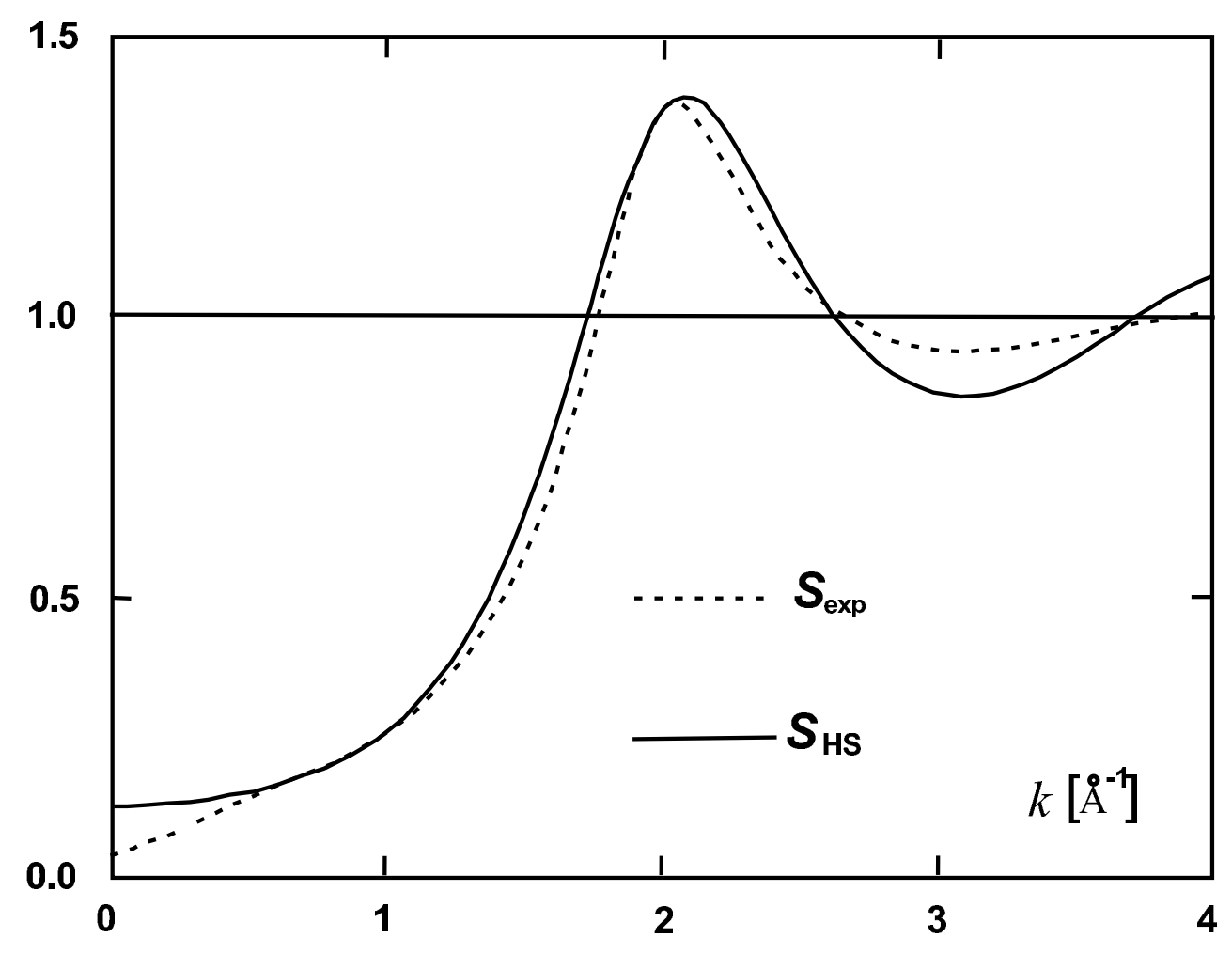

Figure 3: The $k$ - and $T$-dependence of $\Delta S_{\exp }(k ; T)$ is qualitatively reproduced by the calculated result $\Delta S_{\text {theor }}$. The absolute values of $\Delta S_{\text {theor }}$ are, however, too small by a factor 2 to 3 . We used different vertical scales for the two quantities in order to emphasize the common features. 